\title{
Pre-dispersing Process of Montmorillonite by Water and Toluene: Influence on Thermal Properties of Ethylene Vinyl Acetate / Montmorillonite Nanocomposites
}

\author{
Azlin Fazlina Osman ${ }^{1 *}$, Tuty Fareyhynn Mohammed Fitri ${ }^{1}$, Yusrina Mat Daud ${ }^{1}$, Md Rakibuddin ${ }^{2}$ and Supri A. Ghani ${ }^{3}$ \\ ${ }^{1}$ Center of Excellence Geopolymer and Green Technology (CEGeoGTech), School of Materials Engineering, \\ Universiti Malaysia Perlis, Arau, Perlis 02600, Malaysia. \\ ${ }^{2}$ Department of Chemistry, Indian Institute of Technology Kharagpur, India. \\ ${ }^{3}$ Faculty of Engineering Technology, Universiti Malaysia Perlis, Kampus UniCITI, S. Chuchuh, Padang Besar, Perlis, Malaysia.
}

\begin{abstract}
Organanically modified montmorillonite (organo-MMT) was incorporated into ethylene vinyl acetate (EVA) copolymer to improve its thermal stability. A processing procedure, the so called 'predispersing' process of the organo-MMT was introduced prior to melt compounding process of both constituents in order to facilitate the nanofiller dispersion in the EVA matrix. Water and toluene were used as pre-dispersing medium, while magnetic stirring and ultrasonication were utilized as pre-dispersing method. The effects of pre-dispersing medium and method on thermal behaviour of neat EVA and EVA nanocomposites were analysed. It was anticipated that improvement in organo-MMT dispersion would enhance the matrix-nanofiller interactions, thereby the thermal properties of the resultant EVA/MMT nanocomposite. Based on thermal studies by thermogravimetric analysis (TGA), the organo-MMT nanofiller pre-dispersed by ultrasonication in water medium for 2 minutes (MMT(W)2m_u) resulted in most significant thermal stabilizing effect to the EVA copolymer. This was due to the significant improvement in the organo-MMT dispersion when the above mentioned pre-dispersing parameters were employed. Apparently, the result indicates that the high temperature behaviour of the nanocomposite can be affected by the strength of interphase interactions between the matrix and nanofiller which is also influenced by the dispersion of the organo-MMT.
\end{abstract}

\section{Introduction}

Ethylene vinyl acetate (EVA) copolymer is a polymeric material that exhibits softness and flexibility similar to elastomeric materials, yet it can be processed like other thermoplastic [1]. EVA is recyclable, offers excellent optical clarity, barrier properties, low-temperature toughness and stress-crack resistance $[1,2]$. However, to be used as advanced material, further improvement in EVA thermal performance is needed, due to its tendency to thermally degrade under harsh environment and elevated temperatures. The enhancement in EVA thermal performance can be achieved by adding small amount of nanoclay as reinforcing filler $[3,4]$. The resultant 'EVA nanocomposite' can perform greater thermal stability as compared to the neat EVA due to the dispersion of more thermally stable layered silicate material in the host copolymer structure. However, the production of fully exfoliated polymer/nanoclay nanocomposites is crucial to obtain an actual benefit of nanoscale reinforcement in the polymer matrix [5]. Dispersion of nanoclay filler must be optimized to effectively shield the polymer chains during thermal degradation process. Previous studies showed that poor dispersion of MMT clay inhibits significant thermal stabilizing effect of this nanofiller to the host EVA copolymer [3, 4]. In this work, pre-dispersing process of the organically modified montmorillonite (organoMMT) was done prior to melt compounding process with the EVA copolymer. The purpose was to obtain EVA/MMT nanocomposite with well exfoliated and dispersed nanofiller and subsequently improve its thermal stability. Magnetic stirring and ultrasonication were used to pre-disperse the organo-MMT under water or toluene medium. The effect of both pre-dispersing medium and method on thermal properties of neat EVA and EVA nanocomposites was observed and compared.

\section{Materials and Method}

\subsection{Materials}

The EVA copolymer used as matrix material was commercially known as COSMOTHENE EVA H2181. The resin was supplied by the Polyolefin Company (Singapore) Pte. Ltd. The EVA composed of $18 \mathrm{wt} \%$ of vinyl acetate (VA) and $82 \mathrm{wt} \%$ ethylene. The organo modified montmorillonite (organo-MMT), which contains $35-45 \%$ of dimethyl dialkyl (C14-C18) amine

* Corresponding author: azlin@unimap.edu.my 
as an organic surfactant was used as nanofiller. This organo-MMT is commercially known as Nanomer (types 1.44P) by Nanocor, Inc. Distilled water and toluene were used as pre-dispersing medium to allow swelling of the organo-MMT. Toluene (ACS, ISO, Reag.PhEur grade) was supplied by Merck Pty Ltd.

\subsection{Samples Preparation}

The organo-MMT powder was pre-dispersed in two types of liquid medium which were distilled water and toluene in order to allow swelling of nanoplatelets prior to melt compounding with the EVA matrix, The organoclay was prepared as $20 \%$ solution in toluene and distilled water respectively and the suspension was stirred at room temperature by using two methods which were ultrasonication and magnetic stirring. After that, the suspension (in water) was placed in the air circulated oven for 48 hours. The suspension of MMT/toluene was left unattend under fume hood for several hours before being placed in the oven for 24 hours at temperature of $50^{\circ} \mathrm{C}$, to ensure the solvent was fully evaporated. Lastly, the dried organo-MMT powders were grinded and sieved to reduce agglomeration. The EVA nanocomposites were prepared according to the formulation summarized in Table 1. The nanocomposite samples were prepared by melt compounding process of EVA copolymer with constant amount of organo-MMT nanofiller (5 wt\%) at processing temperature of $160^{\circ} \mathrm{C}$ using internal mixer (Brabender plasticoder) machine. Then, by using compression moulding machine, the compound was compressed into $1 \mathrm{~mm}$ thick sheets.

Table 1. Formulation of EVA/MMT nanocomposites

\begin{tabular}{|c|c|c|}
\hline \multicolumn{2}{|c|}{ Nanofiller (Organo-MMT) } & $\begin{array}{c}\text { Nanocomposite } \\
\text { (EVA+5\% MMT) }\end{array}$ \\
\hline Pre-dispersing parameter & Acronym & Acronym \\
\hline None & MMT & EVA+MMT \\
\hline $\begin{array}{c}20 \% \text { Organo-MMT suspension in } \\
\text { toluene pre-dispersed by magnetic } \\
\text { stirrer }\end{array}$ & MMT(TL)_m & EVA+MMT(TL)_m \\
\hline $\begin{array}{c}20 \% \text { Organo-MMT suspension in } \\
\text { water pre-dispersed by magnetic } \\
\text { stirrer }\end{array}$ & MMT(W)_m & EVA+MMT(W)_m \\
\hline $\begin{array}{c}20 \% \text { Organo-MMT suspension in } \\
\text { water pre-dispersed by ultrasonic } \\
\text { probe for 2 minutes }\end{array}$ & MMT(W)2m_u & EVA+ \\
\hline
\end{tabular}

\subsection{Thermogravimetric analysis (TGA)}

TGA was carried out by using TGA Pyris Diamond Perkin Elmer equipment, to study on thermal stability of neat EVA and EVA nanocomposite samples. The weight loss of specimen against temperature was measured from $30^{\circ} \mathrm{C}$ to $700^{\circ} \mathrm{C}$ and with a constant heating rate of $10^{\circ}$ $\mathrm{C} / \mathrm{min}$, under nitrogen atmosphere.

\subsection{Transmission electron microscopy (TEM)}

TEM analysis was carried out using JEOL JEM2010 electron microscope (Japan) operating at $200 \mathrm{kV}$. Prior to viewing, the samples were cut approximately $300 \mathrm{~nm}$ thickness using glass cutter on a Leica Utracut
Ultramicrotome (UCT) instrument at Tg temperature = $80{ }^{\circ} \mathrm{C}$ maintained by using liquid nitrogen.

\section{Results and Discussion}

TGA analysis was conducted to study and compare the thermal stability of neat EVA and EVA nanocomposites containing both pre-dispersed and non-pre-dispersed organo-MMT when heated up to their decomposition temperature. The thermal stability was assessed through the determination of the maximum temperature for degradation $\left(\mathrm{T}_{\mathrm{dmax}}\right)$ of neat EVA and EVA nanocomposites and the rate of mass loss. Table 2 shows the thermal degradation peak temperature of all materials during the first and second step of mass losses, while Figure 1 illustrated TGA curves of the neat EVA and EVA nanocomposites respectively.

Table 2. Thermal degradation peak temperature $\left(T_{d \max }\right)$ of EVA and EVA nanocomposites.

\begin{tabular}{|c|c|c|}
\hline \multirow{2}{*}{ Material } & $1^{\text {st }}$ step mass loss & $2^{\text {nd }}$ step mass loss \\
\cline { 2 - 3 } & $\mathrm{T}_{\mathrm{dmax}}\left({ }^{\circ} \mathrm{C}\right)$ & $\mathrm{T}_{\mathrm{dmax}}\left({ }^{\circ} \mathrm{C}\right)$ \\
\hline EVA & 347 & 497 \\
\hline EVA+MMT & 346 & 492 \\
\hline EVA+MMT(TL)_m & 351 & 523 \\
\hline EVA+MMT (W)_m & 349 & 506 \\
\hline EVA+MMT $(\mathrm{W}) 2 \mathrm{~m} \_\mathrm{u}$ & 355 & 530 \\
\hline
\end{tabular}

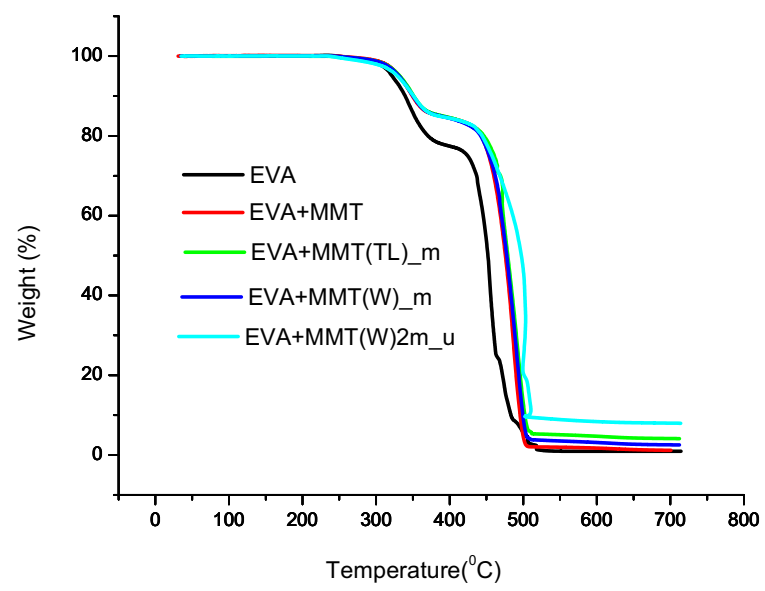

Fig. 1. TGA thermograms of neat EVA and EVA nanocomposites incorporating non-pre-dispersed and predispersed organo-MMTs by various pre-dispersing parameters.

Basically, when subjected to high temperatures, the polymer-filler, filler-filler and filler-aggregate interactions can bring more pronounce effect on the decomposition behaviour of the host polymer, thereby its thermal stability. This is due to the fact that there are larger deviations between the underlying thermodynamics of these distinct materials [3]. High temperature behaviour of the EVA nanocomposite can be much more affected by the strength of interphase interactions between all the components presence in the material. According to Figure 1 and Table 2, the 
decomposition process of EVA is generally occurred in two steps (referred to as first step mass loss and second step mass loss). The first step mass loss between 300 and $400{ }^{\circ} \mathrm{C}$ was due to the deacetylation reaction, in which implicates the releases of gaseous acetic acid and formation of $\mathrm{C}=\mathrm{C}$ along the polymer backbone [3-6]. The second step mass loss in the range between 400 and $600^{\circ} \mathrm{C}$ was attributed to the oxidation and volatilization of hydrocarbons produces from the decomposition of the EVA copolymer backbone [3-6]. The $\mathrm{T}_{\mathrm{dmax}}$ of the first step mass loss of nanocomposites containing EVA+MMT (non-pre-dispersed) occurred at lower temperature as compared to neat EVA. This would possibly due to the catalytic effect from the degradation of the organic surfactant [3-5]. The degradation of dimethyl dialkyl (C14-18) amine, that used to surface modified the MMT accelerated the degradation of polyvinyl acetate (PVA) to acetic acid. When compared with the ones containing the pre-dispersed nanofillers, apparently, this nanocomposite shows lower $\mathrm{T}_{\mathrm{dmax}}$ of the first step mass loss. Poorer EVA-nanofiller interactions may lead to more severe catalytic effect on the deacetylation of EVA during the decomposition process. This is because, weaker interactions between MMT nanoplatetets and the EVA molecular chains provides less shielding for the degradation process of the organic surfactant. As the organic surfactant degrades faster, it will bring stronger catalytic effect to the deacetylation of the EVA. In contrary, the $T_{\mathrm{dmax}}$ of the first step mass loss for all nanocomposites containing the pre-dispersed organo-MMTs shows higher in values compared to neat EVA. This was due to enhanced MMT dispersion (Figure 2) causing greater nanofiller-PVA chains interactions and more restricted polymer chain mobility. Consequently, higher temperature was needed to break the bonds.

The $T_{\text {dmax }}$ values of second step mass loss revealed more significant effect of the pre-dispersing procedure to the thermal stability of the resulting EVA nanocomposites. The $\mathrm{T}_{\mathrm{dmax}}$ of all nanocomposites containing the predispersed nanofillers occur at much higher temperature as compared to neat EVA and EVA+MMT nanocomposite. This shows that the capability of the well dispersed and interacted nanofiller to enhance the thermal stability of the host polymer is more obvious at high temperature decomposition process. In agreement with the statement of Ray \& Okamoto [6], the nanoclays can act as a heat barrier, which can well assist in the formation of char during thermal decomposition of the polymer. Based on Figure 2, the better dispersed and exfoliated nanofiller $(\mathrm{EVA}+\mathrm{MMT}(\mathrm{W}) 2 \mathrm{~m}$ u) shows the greatest thermal stability among all the materials as it exhibits the highest $\mathrm{T}_{\mathrm{dmax}}$ for both first and second step mass losses. In addition, it produced the highest content of char residue ( $4 \mathrm{wt} \%)$ as compared to EVA+MMT (non-pre-dispersed) and other nanocomposites. Without the nanofiller addition, the neat EVA only exhibit less than $1 \mathrm{wt} \%$ residue ash when decomposed. Finally, the findings also revealed that the application of sonic waves in water medium can more efficiently disperse the organo-MMT nanoplatelets than using magnetic stirring in toluene medium. During ultrasonication, the hydrodynamic effect can produce greater implosion energy of the cavitation bubbles which can penetrate in between the organo-MMT interlayers, thereby facilitate the nanoplatelet/nanolayer exfoliation. Consequently, the obtained pre-dispersed nanofiller can more efficiently disperse in the host EVA copolymer and act as thermal stabilizing agent.
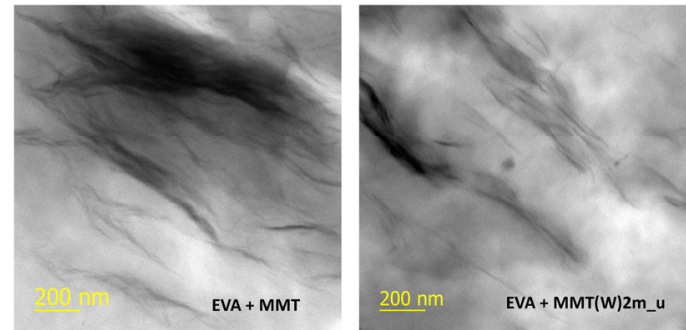

Fig. 2: TEM images of EVA+MMT and EVA+MMT(W) $2 \mathrm{~m} u$ nanocomposites which indicate that the nanocomposite containing the pre-dispersed MMT has greater nanofiller dispersion and smaller tactoid size.

\section{Conclusion}

Based on the TGA results, the MMT(W)2m u is the best nanofiller to enhance the thermal stability of the EVA copolymer. The improved exfoliation and dispersion of the organo-MMT upon the pre-dispersing procedure resulted in enhanced interactions between this nanofiller with the EVA copolymer chains, thereby producing a more thermally stable EVA nanocomposite system.

\section{Acknowledgement}

The authors thank Ministry of Higher Education, Malaysia for funding the project through Fundamental Research Grant Scheme (FRGS) (No: 9003-00473).

\section{References}

1. J. K. Fink, Ethylene Vinyl Acetate Copolymers, in Handbook of Engineering and Specialty Thermoplastics: Polyolefins and Styrenics, John Wiley \& Sons, Inc., Hoboken, NJ, USA, 187209 (2010).

2. A.F. Osman, A.M. Alakrach, H. Kalo, W.N.W Azmi, F. Hashim, RSC Adv., 5, 31485 (2015).

3. A.F. Osman, H. Kalo, M.S. Hassan, T.W. Hong, F. Azmi, , J. of Appl. Polym. Sci., 133, 43204 (2016).

4. A.F. Osman, A.R. Abdul Hamid, Md. Rakibuddin, G.K. Weng, R. Ananthakrishnan, S.A. Ghani, Z. Mustafa, J. of Appl. Polym. Sci., 134, 44640 (2017)

5. A.F. Osman, T.F. Mohammed Fitri, Md. Rakibuddin, F. Hashim, S.A.T.T. Johari, R. Ananthakrishnan, R. Ramli, Materials Science and Engineering C, 74, 194 (2017).

6. S.S Ray, \& M. Okamoto. Polymer/layered, Prog. Polym. Sci., 281539 (2003). 